\title{
DESIGN AND SIMULATION ANALYSIS OF AN ELECTROSTATIC ACTUATOR FOR IMPROVING THE PERFORMANCE OF SCANNING PROBE NANOLITHOGRAPHY
}

\author{
Le Van Tam ${ }^{1}$, Dang Van Hieu ${ }^{1,2}$, Nguyen Duy $\mathrm{Vy}^{3,4}$, Vu Ngoc Hung ${ }^{1}$, \\ Chu Manh Hoang, ${ }^{*}$ * \\ ${ }^{1}$ International Training Institute for Materials Science, Hanoi University of Science and \\ Technology, No. 1, Dai Co Viet, Hai Ba Trung, Hanoi, Viet Nam \\ ${ }^{2}$ Department of Electronics and Communications, Thanh Do University, Hanoi, Viet Nam \\ ${ }^{3}$ Theoretical Physics Research Group, Ton Duc Thang University, Ho Chi Minh City 756636, \\ Vietnam \\ ${ }^{4}$ Faculty of Applied Science, Ton Duc Thang University, Hochiminh City 756636. Vietnam. \\ "Email: hoangcm@itims.edu.vn
}

Received: 22 October 2016; Accepted for publication: 21 February 2017

\begin{abstract}
In this paper, we design and simulate a micro-suspension based scanning probe for nanolithography using electrostatic actuation. The probe consists of a square plate with a pyramid tip at the center that is suspended by four beams. The entire probe is assumed to be made of single silicon and operated in air medium. Operation characteristics are analyzed by finite element method. The operation mode is symmetrical that overcomes the lateral displacement in the unsymmetrical operation mode of conventional scanning probe nanolithography, hence increasing the precision in lithographed nanostructures. The effect of electric field fringe and fixed electrode to the operation of the scanning probe are also analyzed in detail.
\end{abstract}

Keywords: electrostatic actuator, symmetrical operation mode, scanning probe lithography.

\section{INTRODUCTION}

The scanning probe based lithography has been developed to replace the conventional photolithography technology due to disadvantages such as the resolution limited by the diffraction phenomena and the requirement of expensive equipments. In order to fabricate patterns on surfaces with nano-scale dimensions and precision, one can use an atomic force microscope probe coated with a chemical and slowly translated over the surface in contact mode [1 - 5]. 
The microscope scanning probes are not only known as imaging by scanning probe on the sample surface but also used to produce nano-scale patterns. From this point of view, specialized scanning probes have been researched and developed for nanostructure fabrication. The scanning probes usually use cantilever structure in which a probe is integrated at the free end of a silicon fixed-free beam. This structure can be controlled by three actuation methods such as: thermal actuation method, piezoelectric actuation method, and electrostatic actuation method [6 - 8]. The operation mode of scanning probe is unsymmetrical, which limits the controllable precision of lithographed structures. We proposed a fixed-fixed beam structure with an electrostatic actuator for improving the limitation in lithography process causing by the unsymmetrical operation mode of cantilever-type [9]. Comparing with cantilever beam structure, at same size, stiffness of fixed-fixed structure is lager, pull-in voltage increases significantly. However, the fixed-fixed beam structure can't fabricate tip array on a probe.

In this paper, we propose a symmetrically operated electrostatic actuator to increase the accuracy in nanolithography by suppressing lateral displacement. Due to the rectangular plate has high hardness compared to the suspending beams, so it does not be bent when impacted. We also investigated fringe field effect by changing the area of the fixed plate. In addition, electrostatic force also makes the beam stiffness alter, which causes the displacement of the beam and capacitance changed. Compared to the fixed-fixed beam structure, the capacitance of actuator is also increased. When actuated, the plate displaces parallelly to the sample surface, so we can integrate a tip array on the plate and increasing throughput nanolithography [10]. Moreover, with micro-suspension structure we can adjust operating frequency more flexible.

\section{DESIGN OF MICRO-SUSPENSION STRUCTURE}

In conventional design, the scanning probe is often based on cantilever-like structure as shown in Fig. 1 (a). When the cantilever is actuated in vertical direction, there is a lateral displacement. This lateral displacement is significant, when the amplitude of vertical actuation is large. This effect can cause difficulty for control, even error in lithography process at nano-scale. Taking this effect into considering, we propose an electrostatic actuator for improving the performance of scanning probe nanolithography.

A schematic drawing of the proposed micro-suspension electrostatic actuator is shown in Fig. 1 (b). A square plate with a pyramid tip at the center is suspended by four simple beams, and the whole structure is assumed to be made of single crystal silicon. In order to actuate the probe there is a counter-electrode in parallel with the back side of the probe. To analyze the structure, several assumptions are used:

- Single crystal silicon is isotropic material that has the same properties in all directions.

- The bending of beams conforms to Euler-Bernoulli beam theory.

- The plate is a rigid body in which deformation is neglected.

The natural frequencies of the structure are calculated by lumped-parameter method. Each beam is transformed into a linear mass-spring with equivalent stiffness $k_{e q}$, equivalent mass $m_{e q}$ and connected in parallel, shown in Fig. 2. When an applied force $F$ on the end of the beam, the deflection curve of the beam $v(x)$ is derived by solving four-order ordinary differential equation of beam [11 - 12].

$$
E I \frac{d^{4} v(x)}{d x^{4}}=0
$$


The boundary conditions of the fixed-slide beam are:

$$
v(0)=0, v^{\prime}(0)=0
$$

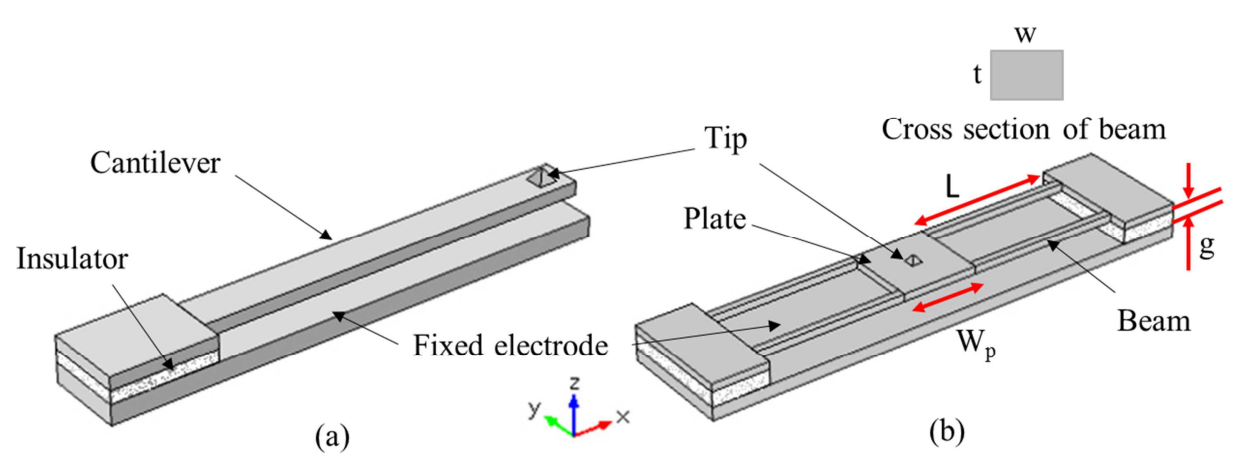

Figure 1. Structure of the conventional scanning probe (a) and the proposed micro-suspension electrostatic actuator (b).

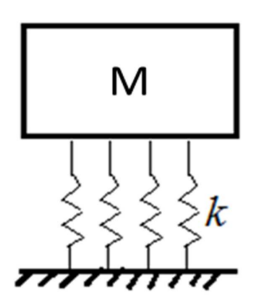

Figure 2. Equivalent Lumped-parameter model of the micro-suspension electrostatic actuator.

$$
v(L)=z, v^{\prime}(\mathrm{L})=0
$$

where, $E$ is the Young's modulus, $I$ is the cross-sectional moment of area with respect to axes, $L$ length of the beam, and $\mathrm{z}$ is the vertical displacement of probe. The deflection curve and maximum deflection of the beam are:

$$
\begin{gathered}
v(x)=\frac{F}{12 E I}\left(3 L x^{2}-2 x^{3}\right) \\
v(L)_{\max }=\frac{F L^{3}}{12 E I}
\end{gathered}
$$

From (2.3), the equivalent stiffness of beam along $\mathrm{y}$ and $\mathrm{z}$ directions are:

$$
k_{e q}^{z}=\frac{12 E I_{y y}}{L^{3}}, \quad k_{e q}^{y}=\frac{12 E I_{z z}}{L^{3}}, \text { respectively. }
$$

The equivalent mass of beam is derived by using Rayleigh method that is calculated from equation [13]:

$$
m_{e q}=\int_{L} \rho A(x) f^{2}(x) d x
$$


where, $\rho$ is mass density, $A(x)$ is cross-sectional area of beam. $f(x)\left(=\frac{\nu(x)}{v_{\max }}\right)$ is bending distribution function. Solving (2.5) we obtain:

$$
m_{e q}=\frac{13}{35} m
$$

where $m$ is the mass of beam.

The natural frequency of structure can be calculated by

$$
f_{e q}=\frac{1}{2 \pi} \sqrt{\frac{K}{M}},
$$

where $K=4 k_{e q}, M=4 m_{e q}+m_{\text {plate }}$ and $m_{\text {plate }}$ is the mass of plate.

The natural frequency of the first order mode (vertical flexural mode) is

$$
f_{1 s t}^{z}=\frac{1}{\pi} \sqrt{\frac{k_{e q}^{z}}{4 m_{e q}+m_{\text {plate }}}}
$$

The natural frequency of the second order mode $f_{2 n d}^{y}$ (lateral flexural mode) is

$$
f_{2 n d}^{y}=\frac{1}{\pi} \sqrt{\frac{k_{e q}^{y}}{4 m_{e q}+m_{\text {plate }}}}
$$

The probe resonantly operates in the first order mode, because the natural frequencies of the first order mode and the second order mode are discrepant enough to prevent mechanical coupling between two modes. The discrepancy between the natural frequencies of two modes $\delta_{f}$ is defined as:

$$
\delta_{f}=100\left[\frac{f_{2 n d}^{y}-f_{1 s t}^{z}}{f_{1 s t}^{z}}\right]
$$

By substituting (2.8) and (2.9) into (2.10) $\delta_{f}$ becomes:

$$
\delta_{f}=100\left[\frac{w}{t}-1\right]
$$

The displacement of the tip is measured by capacitance change. The capacitance of structure $C$ consists of parallel capacitance $C_{\text {parallel }}$ and capacitance caused by fringe effects $C_{\text {fringe, }}$ which is calculated by

$$
\begin{gathered}
C=C_{\text {parallel }}^{\text {plate }}+C_{\text {fringe }}^{\text {plate }}+4\left(C_{\text {parallel }}^{\text {beam }}+C_{\text {fringe }}^{\text {beam }}\right) \\
C_{\text {parallel }}=\frac{\varepsilon_{0} S}{g}
\end{gathered}
$$




$$
C_{\text {fringe }}=C_{\text {parallel }}\left\{1+\frac{2 g}{\pi w} \ln \frac{\pi w}{g}+\frac{2 g}{\pi w} \ln \left[1+\frac{2 t}{g}+2 \sqrt{\frac{t}{g}+\frac{t^{2}}{g^{2}}}\right]\right\}
$$

where, $\varepsilon_{o}$ is the dielectric constant of medium, $S$ is the area of electrodes, and $g$ is the gap between the two electrodes.

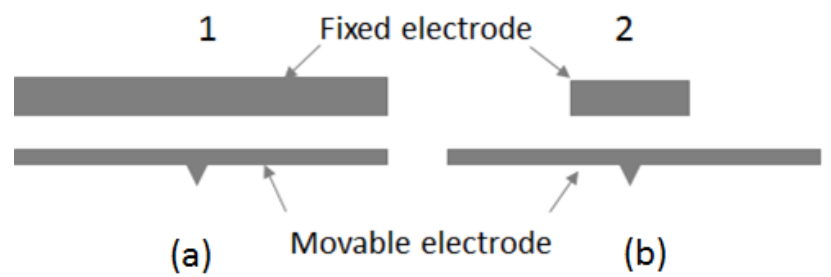

Figure 3. (a) The fixed electrode area is equal to the entire back side area of the probe. (b) The fixed electrode area is equal to the backside area of the plate.

Here, fringe capacitance can be caused fringing fields by the circumference of the plate and spring beams. There are two cases used for actuating the probe. The case 1, the counter electrode is placed on the whole structure as shown in Fig. 3 (a). In this case, $C_{\text {fringe }}$ is caused not only by the fringing field by the plate but also the spring beams. The case 2 , the counter electrode is directly placed only on the plate (Fig. 3 (b)), so the $C_{\text {fringe }}$ is only caused by fringing field around the circumference of the plate.

Table 1. Parameters of the micro-suspension.

\begin{tabular}{llll}
\hline & Length & Width & Height \\
\hline Beam & $300 \mu \mathrm{m}$ & $7 \mu \mathrm{m}$ & $5 \mu \mathrm{m}$ \\
\hline Plate & $100 \mu \mathrm{m}$ & $100 \mu \mathrm{m}$ & $5 \mu \mathrm{m}$ \\
\hline Tip & $14 \mu \mathrm{m}$ & $14 \mu \mathrm{m}$ & $10 \mu \mathrm{m}$ \\
\hline
\end{tabular}

From the above analysis, we calculate, design, and simulate a micro-suspension scanning probe with parameters shown in Table 1. The designed gap between the two electrodes is $2 \mu \mathrm{m}$. The simulation of scanning probe is carried out by finite element method (FEM) in Comsol Multiphysics. Comsol Multiphysics is a powerful interactive environment for modeling and solving all kinds of scientific and engineering problems. Especially, Comsol Multiphysics provides a MEMS module for modeling and simulating MEMS devices such as actuator, sensor oscillators, filters and so on.

\section{RESULTS AND DISSCUSION}

Figure 4 shows the vertical flexible mode shape of the scanning probe by FEM. The operation frequency of the first mode is $56.662 \mathrm{kHz}$, while the second mode is $86.612 \mathrm{kHz}$ and the discrepancy between the two modes $\delta_{f}$ is calculated to be $46 \%$. Using the above parameters, we also calculated the natural frequencies of the first and second mode are 
$f_{1 s t}^{z}=60.432 \mathrm{kHz}$ and $f_{2 n d}^{y}=84.605 \mathrm{kHz}$, respectively, so, the discrepancy between the nature frequency of two modes $\delta_{f}$ is $40 \%$. The analytical results are suitable to the FEM results. The error between the calculation and simulation is defined as \% error $=100\left[\frac{f_{\text {fem }}-f_{a}}{f_{a}}\right]$, the errors

for the first and second mode are $-6.2 \%$ and $-2.4 \%$, respectively. The first mode error is higher because the plate is stiffer in the lateral flexible mode, which is more consistent with assumed rigid body.

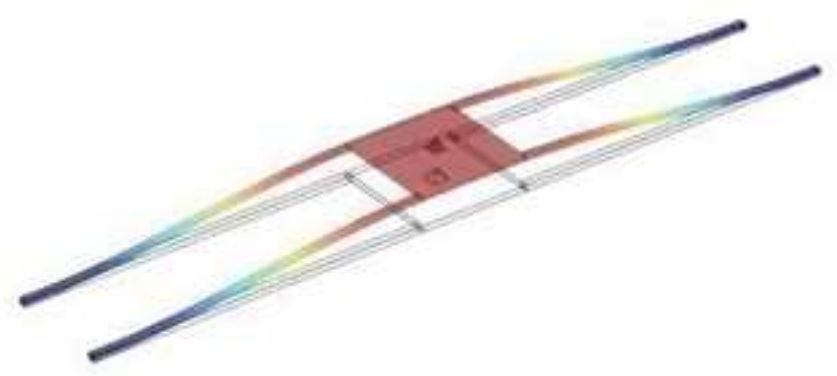

Figure 4. 3D view of the first operation mode of the scanning probe.

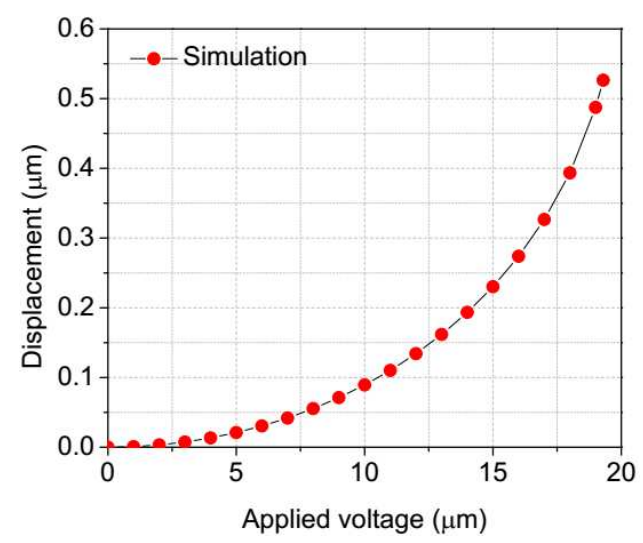

Figure 5. The vertical displacement of the scanning probe tip investigated as a function of applied voltage for case 1 .

The displacement of the tip is controlled by applied voltage. For the case 1, the displacement of tip dependent on applied voltage is investigated and shown in Fig. 5. For electrostatic actuation, the scanning probe will be crashed into sample surface if the displacement is larger than $g / 3$. This is due to pull-in effect occurring in the parallel-plate electrostatic actuation [14]. Therefore, we ensure that the scanning probe is operated in the safe region in all time by using suitable operation voltage. For this structure, when applied voltage is about $19 \mathrm{~V}$, the displacement is about $5.2 \mu \mathrm{m}$ nearby the unsafe region.

In order to integrate the scanning probe into the controllable electronic circuit, the capacitance of actuator has to be large enough. The dynamic capacitance of structure dependent on applied voltage is shown in Fig. 6. For this actuator, the capacitance changes from $126 \mathrm{fF}$ to $149 \mathrm{fF}$. The calculated result at $g=2 \mu \mathrm{m}$ is $118 \mathrm{fF}$. Comparing with simulation result above, the 
error is $-6.8 \%$. The increase in capacitance consistent with two attracted electrodes. The capacitance is larger compared to the fixed-fixed beam type-actuator, with the same area of electrode. Because the area of side wall is larger, the capacitance caused by fringe effects is larger.

In order to consider the effect of electric field fringe and fixed electrode to the operation of the scanning probe, we have investigated the two cases of actuation electrodes as shown in Fig. 3. The effect of fixed electrode area on the structure is shown in Figs. 7 and 8. In the both cases,

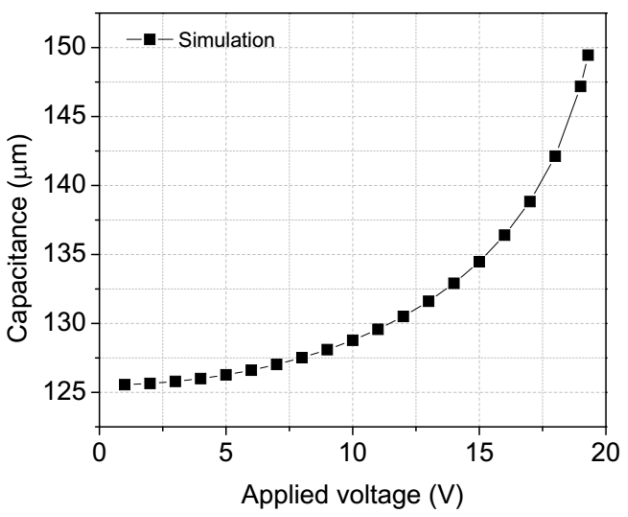

Figure 6. The parallel-plate actuator capacitance of the scanning probe simulated as a function of applied voltage for the case 1 .

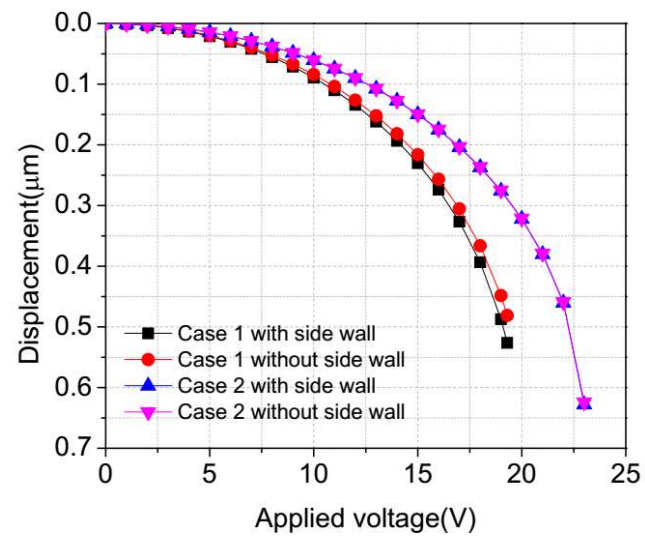

Figure 7. The displacement of tip depends on applied voltage at different plate widths. In the case 1, the fixed electrode area is equal to the entire back side area of the probe. In the case 2 , the fixed electrode area is equal to the back side area of the plate.

the dimensions of the beams are fixed and the width of plate is $100 \mu \mathrm{m}$. In Fig. 7, at the same value of applied voltage, the displacement of tip in the case 1 is larger than in the case 2 . Since the electrostatic force exerting on the probe in the case 1 is larger. In other word, the electrostatic force reduces the stiffness of the beams. In addition, due to the large side wall area, effect of the electric field is significant in the case 1 . The displacement of the tip is significantly reduced when no voltage is applied to the side wall of the probe.

In the case 1, there are additional capacitances between beams and fixed electrode and due to the fringe effect, the capacitance of structure is larger than for the case 2, shown in Fig. 8. 
Additional capacitance due to fringe field is significant in the case 1, so it becomes important to calculate total capacitance of the structure. When the displacement changes, the capacitance disparity between the two cases is insignificant. Since change in additional capacitance is slight. Change in capacitance between a beam and the fixed electrode is investigated as a function of displacement at the end of the beam shown in Fig. 9. For a displacement about $0.51 \mu \mathrm{m}$, the change in capacitance is about $1.5 \mathrm{fF}$. The capacitance depends linearly on the displacement. In the case 2, the capacitance at the gap of $2 \mu \mathrm{m}$ is $46 \mathrm{fF}$ in simulation and $48 \mathrm{fF}$ in calculation, the error between calculation and simulation is $4.1 \%$. In order to select the appropriate capacitance for controlling the probe, we investigated the dependence of capacitance on the plate width. Fig. 10 shows the capacitance values in the two cases with the change in plate width at the constant gap of $2 \mu \mathrm{m}$. With a plate width of $500 \mu \mathrm{m}$, capacitance value in both cases is larger $1 \mathrm{pF}$ and depends primarily on the plate area.

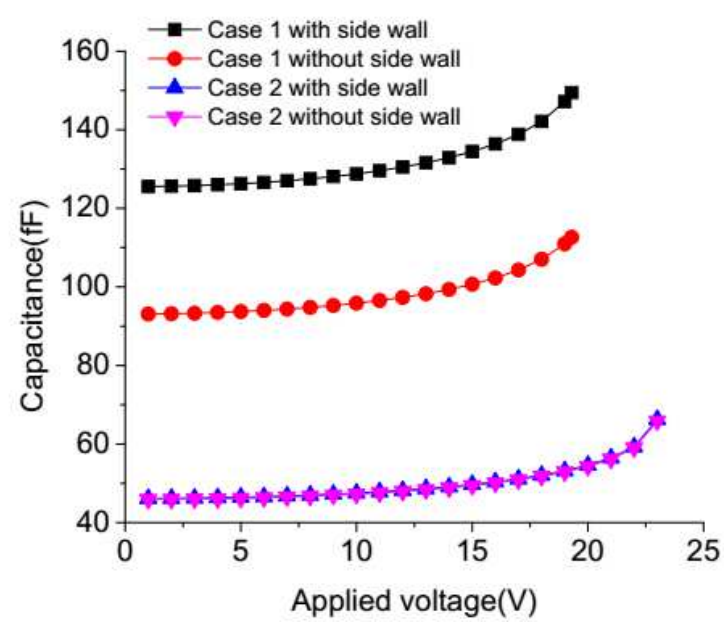

Figure 8. The capacitance of structure investigated as a function of the displacement with different plate widths. In the case 1, the movable electrode area is the entire back side of the probe. In the case 2, the movable electrode area is the back side of the plate.

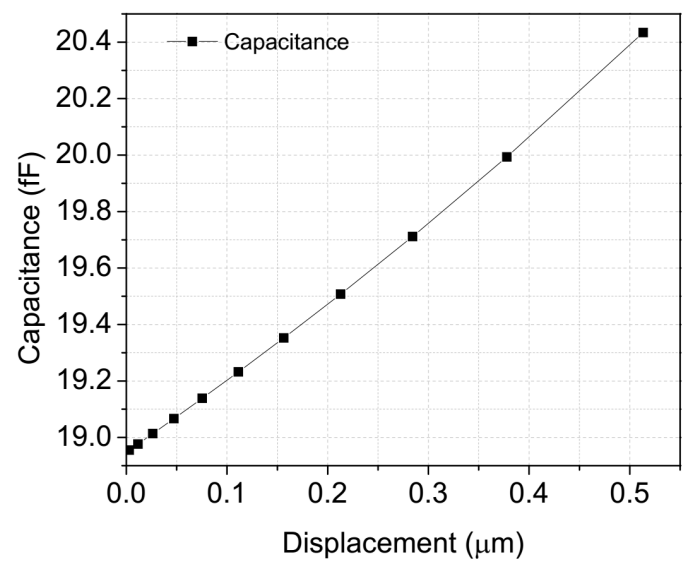

Figure 9. Capacitance between a beam and fixed electrode investigated as a function of displacement at the end of the beam. 


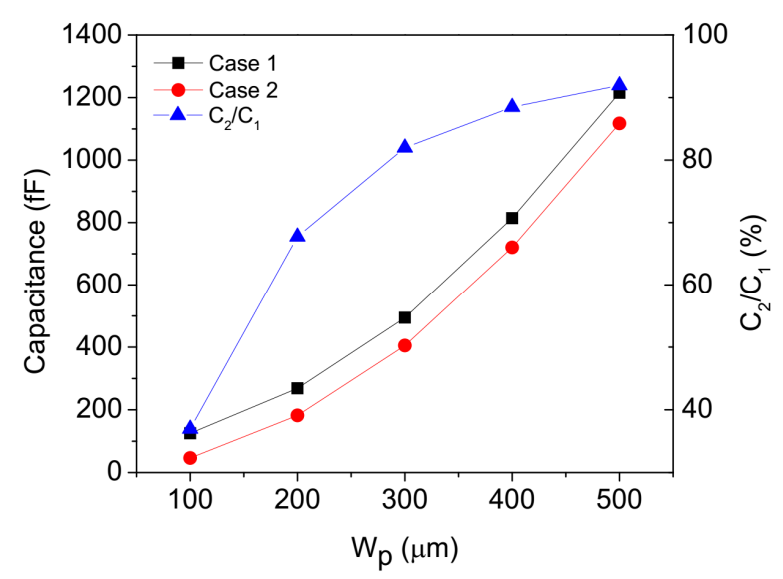

Figure 10. Capacitance of structure at the gap of $2 \mu \mathrm{m}$ forthe case 1 with capacitance $\mathrm{C}_{1}$ (line with black symbol), for the case 2 with capacitance $C_{2}$ (line with red symbol), and the capacitance ratio $C_{2} / C_{1}$.

\section{CONCLUSIONS}

We reported on a symmetrically operated electrostatic actuator for improving the performance of scanning probe lithography. An analytical approach to evaluate the natural frequencies and capacitance of the probe was presented and verified by finite element method. The operation characteristics wee analyzed by using finite element method, which werecompared with the fixed-fixed beam-type actuator. The effect of electric field fringe on the operation of scanning probe was also investigated. A scanning probe structure with actuation capacitance larger than $1 \mathrm{pF}$ can be realized by choosing suitable designing parameters.

\section{REFERENCES}

1. Ginger D. S., Zhang H. - The evolution of dip-pen nanolithography, Angew.Chem. Int. Ed. 43 (2004) 30-45 [doi: 10.1002/anie.200300608].

2. Thomas P. J., Kulkarni G. U., Rao C. N. R. - Dip-pen lithography using aqueous metal nanocrystal dispersions, J. Mater. Chem. 14 (2004) 625-628 [doi: 10.1039/B311248A].

3. Liu X., Li Y., Zheng Z. - Programming nanostructures of polymer brushes by dip-pen nanodisplacement lithography (DNL), Nanoscale 2 (2010) 2614-2618 [doi: $10.1039 / \mathrm{c} 0 \mathrm{nr} 00565 \mathrm{~g}]$.

4. Irvine E. J., Santana A. H., Faulds K., Graham D. - Fabricating protein immunoassay arrays on nitrocellulose using Dip-pen lithography techniques, Analyst 136 (2011) 2925 [doi: 10.1039/c1an15178a].

5. Son J. Y., Shin Y. H., Ryu S., Kim H., Jang H. M. - Dip-Pen Lithography of Ferroelectric PbTiO3 Nanodots, J. Am. Chem. Soc. 131 (2009) 14676-14678 [doi: 10.1021/ja906871b].

6. Wang X., Bullen D. A., Zou J., Liu C., Mirkin C. A. - Thermally actuated probe array for parallel dip-pen nanolithography, J. Vac. Sci. Technol. B 22 (6) (2004) 2563-2567 [http://dx.doi.org/10.1116/1.1805544].

7. Iloh T., Ohashi T., Siiga T. - Piezoelectric cantilever array for multiprobe scanning force microscopy, Proceedings: IEEE micro electro mechanical systems workshop, 1996, pp. 451-455 [doi: 10.1109/MEMSYS.1996.494024]. 
8. Bullen D., Liu C. - Electrostatically actuated dip pen nanolithography probe arrays, Sens. Actuators. A 125 (2006) 504-511 [http://dx.doi.org/10.1016/j.sna.2005.09.001].

9. Tam L. V., Hieu D. V., Hung V. N., Hoang C. M. - Electrostatic Actuator for Improving Scanning Probe Lithography, 9th Vietnam National Conference of Solid Physics and Materials Science, 2015.

10. Eichelsdoerfer D. J., Liao X., Cabezas M. D., Morris W., Radha B., Brow K. A., Giam L. R., Braunschweig A. B., Mirkin C. A. - Large-area molecular patterning with polymer pen lithography. Nat. Protoc. 8 (2013) 2548-2560 [doi:10.1038/nprot.2013.159].

11. Gere J. M., Timoshenko S. P. - Mechanics of Materials, PWS Publishing Company, 1997, 688-690 [ISBN: 0534921744].

12. Urey H., Kan C., Davis W. O. - Vibration mode frequency formulae for micromechanical scanners, J. Micromech. Microeng. 15 (2005) 1713-1721 [http://dx.doi.org/10.1088/09601317/15/9/013].

13. Lobontiu N. - Dynamics of Microelectromechanical Systems, Springer Science, 2007, pp. 9-10 [ISBN: 978-0-387-36800-9].

14. Zhang W. M., Yan H., Peng Z. K., Meng G. - Electrostatic pull-in instability in MEMS/NEMS: A review, Sens. Actuators. A 214 (2014) 187-218.

[http://dx.doi.org/10.1016/j.sna.2014.04.025]. 